\title{
WIDE SEPARATION LENSES
}

\author{
D. J. MORTLOCK AND R. L. WEBSTER \\ University of Melbourne, \\ Parkville, Victoria, 3052, Australia \\ AND \\ P. C. HEWETT \\ Institute of Astronomy, \\ Madingley Road, Cambridge, CBз OHA, UK
}

There is still debate as to whether wide separation $\left(\Delta \theta \gtrsim 3^{\prime \prime}\right)$ double quasars are physical binaries or gravitationally lensed sources. We proceed under the assumption that most of these objects are the result of lensing, and use maximum likelihood techniques (Kochanek 1993) to infer information about the mass distribution of deflectors that would be required to produce these wide image separations. Under the above assumptions, the most consistent explanation is the existence of a significant population of dark objects with the mass of groups of galaxies.

About half of all candidate lens pairs have wide separations, and, as such, cannot be the result of gravitational lensing by a galaxy. Since the Einstein radius of clusters is of the order of $20^{\prime \prime}$ (much larger than any of the image separations), we propose a population of group sized masses as the main deflectors. In this case, these deflectors must be very dark, as few of the quasar pairs have a visible lens between them. This picture of groups as being mostly composed of dark matter fits in well with some recent observations (e.g., Mulchaey et al. 1993).

We use the $(\Lambda=0)$ cosmologies of Turner et al. (1984), and model the lenses as singular isothermal spheres (characterized by $\sigma$, their line of sight velocity dispersion). Their number density is modeled by a broken power law, with a form similar to the Schechter function for low $\sigma$, but with a shallower falloff for the higher masses. Explicitly, we take

$$
\frac{d n}{d \sigma}= \begin{cases}\frac{n_{*}}{\sigma_{*}}\left(\frac{\sigma}{\sigma_{*}}\right)^{-4} & \text { for } \quad \sigma<\sigma_{*} \\ \frac{n_{*}}{\sigma_{*}}\left(\frac{\sigma}{\sigma_{*}}\right)^{\beta} & \text { for } \quad \sigma \geq \sigma_{*},\end{cases}
$$




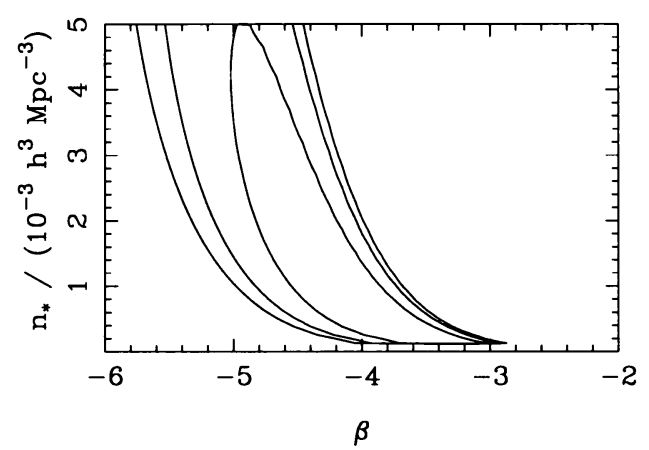

Figure 1. The maximum likelihood results shown in the $\beta-n_{*}$ plane. The contours are at the $68 \%, 95 \%$ and $99 \%$ (i.e., the 1,2 and 3 standard deviation) confidence levels. The calculation implies that $\beta=-4 \pm 1$ and $n_{*}=(3 \pm 3) \times 10^{-3} h^{3} \mathrm{Mpc}^{-3}$.

where $\beta$ and $n_{*}$ are allowed to vary.

To constrain the parameters of the model, we used the Large Bright Quasar Survey (LBQS) (Hewett et al. 1995), a sample of 1055 quasars, with $16.0<B_{J}<18.85$. Hence this sample will not include many reddened objects, and may thus be biased against lensing events (Webster et al. 1995). The LBQS contains three lens pairs, of angular separations $1.48^{\prime \prime}$ (Hewett et al. 1994), 5.14" (Hewett et al. 1989) and 7.5" (unpublished).

Figure 1 shows the best fit to the maximum likelihood calculation as $\beta \approx-4$ and $n_{*} \approx 3 \times 10^{-3} h^{3} \mathrm{Mpc}^{-3}$. Some cosmological simulations imply the number density of clusters is proportional to $M^{-2} \propto \sigma^{-4}$, so our results suggest that number density may vary in this fashion from the scale of galaxies all the way up to clusters. However, the selection effects in the LBQS and the small number statistics must be more carefully considered before these conclusions can be stated with confidence; this work will be presented more fully elsewhere.

\section{References}

Hewett, P.C., Foltz, C.B. \& Chaffee, F.H., 1995, AJ, 109, 1498

Hewett, P.C., Irwin, M.J., Foltz, C.B., Harding, M.E., Corrigan, R.T., Webster, R.L. \& Dinshaw, N., 1994, AJ, 108, 1534

Hewett, P.C., Webster, R.L., Harding, M.E., Jedrzejewski, R.I., Foltz, C.B., Chaffee, F.H., Irwin, M.J. \& Le Févre, O., 1989, ApJL, 346, L61

Kochanek, C.S., 1993, ApJ, 419, 12

Mulchaey, J.S., Davis, D.S., Mushotzky, R.F. \& Burstein, D., 1993, ApJL, 404, L9

Turner, E.L., Ostriker, J.P. \& Gott, J.R., 1984, Astrophysical Journal, 284, 1

Webster, R.L., Francis, P.J., Peterson, B.A., Drinkwater, M.J. \& Masci, F.J., 1995, Nature, 375,469 